Background: The authors present a patient who developed adenocarcinoma within the ileal "J" pouch-anal anastomosis (IPAA) after restorative proctocolectomy for familial adenomatous polyposis (FAP).

Case report: The patient was treated for recurrent adenomatous polyps within the ileoanal anastomosis.

Regular examination of the patient with endoscopic, histopathological and radiological measures such as ultrasonography (USG), computed tomography (CT) and magnetic resonance imaging (MRI) did not reveal malignant transformation in recurrent adenomatous polyps. Only positron emission tomography/computed tomography (PET/CT) showed a suspicious lesion. As a result abdominoperineal excision of the ileal pouch and anal canal was performed and diagnosis of cancer was confirmed on histopathological assessment.

Conclusion: In our opinion PET/CT is recommended in each case of dysplasia in patients operated on for FAP.

Key words: familial adenomatous polyposis, restorative proctocolectomy, positron emission tomography/computed tomography.

\section{PET/CT in recognition of a complicated case of adenocarcinoma within ileal pouch-anal anastomosis in a patient after restorative proctocolectomy for familial adenomatous polyposis}

Tomasz Banasiewicz ${ }^{1}$, Jacek Paszkowski ${ }^{1}$, Rafał Czepczyński ${ }^{2}$, Jacek Hermann ${ }^{1}$, Andrzej Pławski ${ }^{3}$, Jacek Szmeja ${ }^{1}$, Michat Drews ${ }^{1}$

1Department of General, Gastrointestinal and Endocrinological Surgery, Poznan University of Medical Sciences, Poznań, Poland

2Department of Endocrinology, Poznan University of Medical Sciences and PET/CT Centre, Euromedic Diagnostics, Poznań, Poland

Institute of Human Genetics, Polish Academy of Sciences, Poznań, Poland

\section{Background}

Restorative proctocolectomy is indicated in patients with familial adenomatous polyposis (FAP) if multiple polyps occur in the rectum and reach the dentate line of the anal canal. Anyway, the patients still are put at risk of developing polyps in the ileal pouch and within the anal transitional zone epithelium [1]. Therefore, regular endoscopic examination and radiological measures such as USG, CT and MRI are recommended in those patients after the surgical treatment [2].

\section{Case report}

The patient I.D., age 48, was admitted to the clinic due to suspicion of cancer within the ileal "j" pouch-anal anastomosis after restorative proctocolectomy for familial polyposis which was performed in 1992. FAP was diagnosed in the patient at the age of 31 on the basis of endoscopic and histopathological assessment which showed multiple adenomatous polyps located in the rectum and sigmoid colon. History revealed FAP in 5 members of the patient's family. In addition, substitution of c.2626C $>T$ in codon 876 CGA $>$ TGA, R>X (arginine > STOP) was found on genetic examination. The patient was seen regularly after the primary operation for periods from half to one year and he underwent numerous endoscopic polypectomies for recurrent polyps located within the ileal-anal anastomosis and the anal transitional zone epithelium. Histopathological examination showed adenomatous polyps with low grade dysplasia. In addition, signs of severe, recurrent chronic pouchitis and anal sphincter damage occurred in the patient. Finally, diverting loop ileostomy was performed due to increasingly severe diarrhoea and faecal incontinence. Diffuse inflammatory infiltration within the ileal pouch was recognized during laparotomy. Inflammation was also diagnosed on USG, $\mathrm{CT}$ and MRI.

Afterwards, additional polypectomies were performed revealing tubular and villous adenomas. Because the patient was in a good general condition and he was reluctant to undergo another difficult operation, and there was no malignancy in any examination method, the idea of abdominoperineal pouch excision was abandoned. Anyway, PET/CT was done which revealed infiltration within the ileal pouch-anal anastomosis 22/39 $\mathrm{mm}$ in diameter with pathological 18-fluorodeoxyglucose uptake $\left(S U V_{\text {max }} ;{ }^{18} \mathrm{~F}-\mathrm{FDG}=13.2\right.$ ) suggesting malignancy (Fig. 1). As a result, the patient and doctors made the 


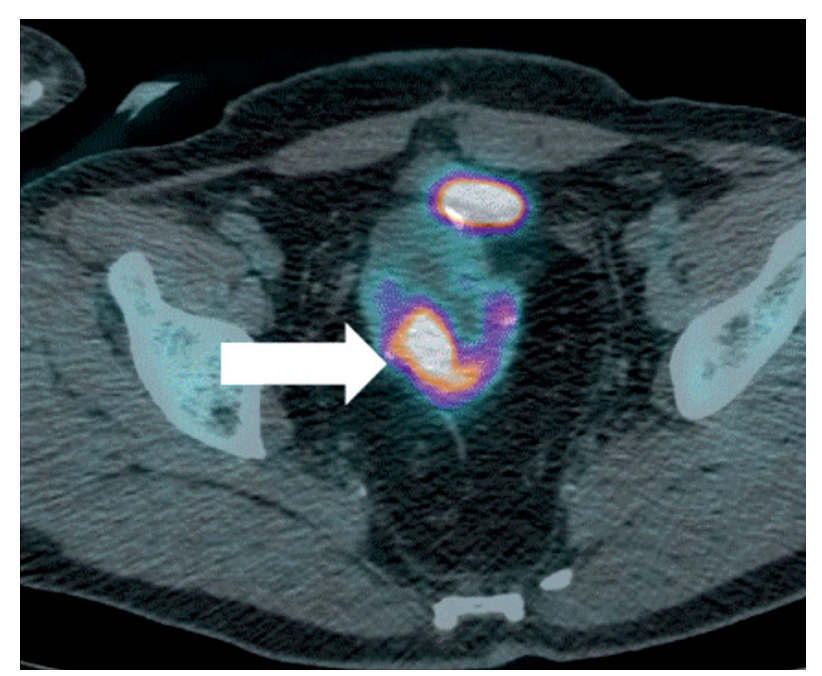

Fig. 1. PET/CT - the arrow indicates a lesion of pathological uptake of $18 \mathrm{~F}-\mathrm{FDG}$, recognized as cancer. Filled urinary bladder above

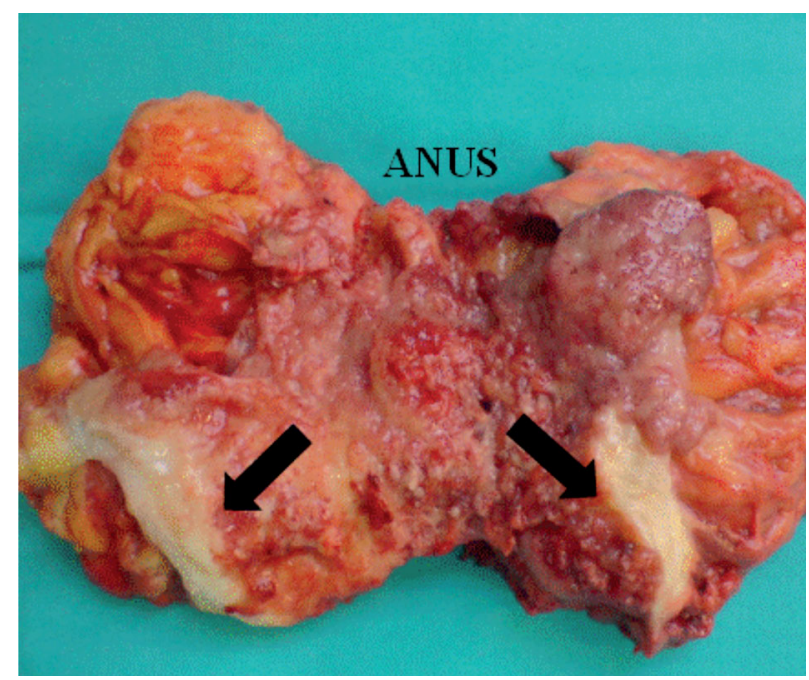

Fig. 3. Specimen of excised anal canal with visible lesion of cancer, localized intramurally (arrows) and multiple adenomatous polyps with dysplasia foci

decision to perform abdominoperineal excision of the ileal pouch and anal canal in two stages. During the first procedure the pouch with distal ileal loop was removed and terminal ileostomy performed (Fig. 2). Perineal excision of the anal canal with sphincters was accomplished as a second step operation. Histopathological examination of the specimen showed adenocarcinoma of the ileal-anal anastomosis (G2, T2NOMO) and multiple adenomatous polyps of the distal part of the pouch and anal canal with low and high grade dysplasia (Fig. 3).

\section{Discussion}

Endoscopic examination with radiological measures such as USG, CT and MRI is recommended for surveillance in patients undergoing restorative proctocolectomy with ileal pouch-anal anastomosis for FAP in order to recognize precancerous conditions as well as malignant lesions located within the pouch and anal canal [1]. Standard examination with additional studies were not adequate in the above-

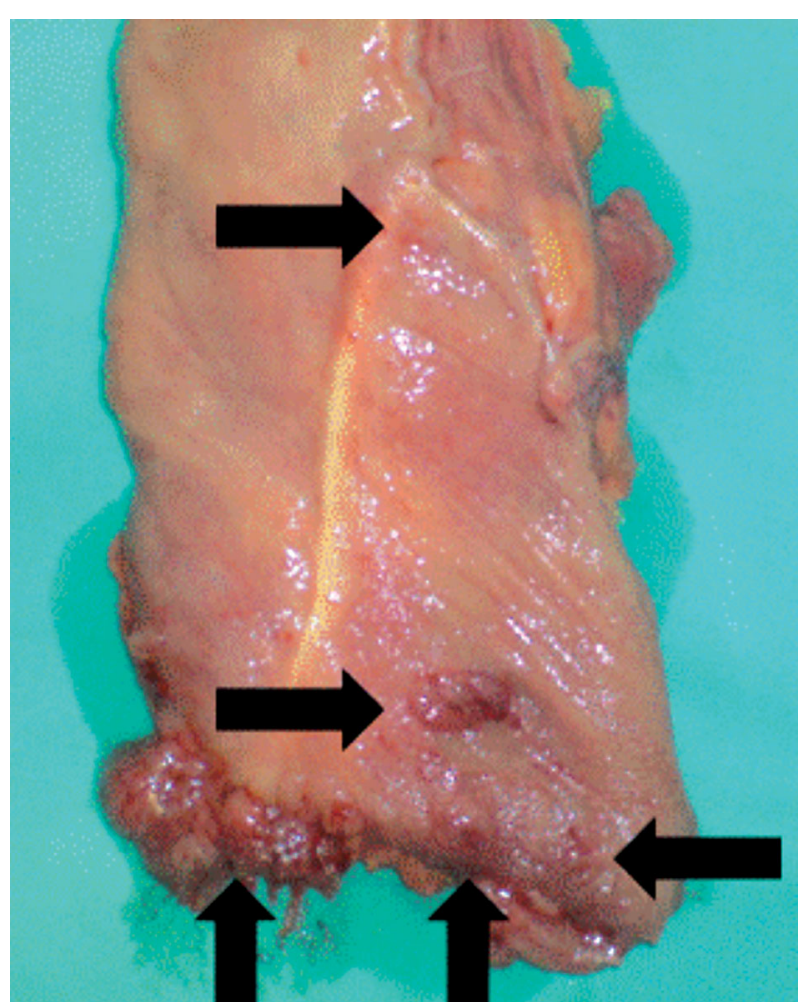

Fig. 2. Specimen of excised ileal "y" pouch with multiple polyps (arrows) with recognized lesions of low and high grade dysplasia on histopathological assessment

mentioned patient. Only PET/CT showed a highly suspicious lesion. The cancerous tissue was localized mainly intramurally within the inflammatory infiltration of the thickened intestinal wall of the ileal-anal anastomosis region. The decision to perform the high-risk operation was made by the patient as well as doctors after analysis of the PET/CT result. The number of patients with FAP in Europe ranges from 11300 to 37600 [3] and only some of them undergo either colectomy or proctocolectomy. The rate of dysplasia after IPAA can reach $18.5 \%$ according to a representative meta-analysis including 2040 patients [4].

Based on the experience with the presented case as well as with other cases described elsewhere [5], we strongly recommend performing PET/CT in each case of dysplasia and in recurrent adenomatous polyps in patients operated on for familial polyposis to improve early diagnosis of malignant lesions.

\section{References}

1. Hurlstone DP, Saunders BP, Church JM. Endoscopic surveillance of the ileoanal pouch following restorative proctocolectomy for familial adenomatous polyposis. Endoscopy 2008; 40: 437-42.

2. Half E, Bercovich D, Rozen P. Familial adenomatous polyposis. Orphanet J Rare Dis 2009; 4: 22.

3. European Medicines Agency Doc. Ref.: EMEA/COMP/264/04draft [http://www.emea.europa.eu/pdfs/human/comp/opinion/02 6404en.pdf]

4. Scarpa M, van Koperen PJ, Ubbink DT, Mommes DW, Ten Kate FJ, Bemelman WA. Systematic review of dysplasia after restorative proctocolectomy for UC. Br J Surg 2007; 94: 534-45. 
5. Czepczyński R, Banasiewicz T, Paszkowski J, Guzikowska-Ruszkowska I, Drews M, Sowiński J. PET/CT using 18F-FDG in patients with familial adenomatous polyposis - preliminary report (abstract). Eur J Nucl Med Mol Imaging 2009; 36: S338.

\section{Address for correspondence}

\section{Tomasz Banasiewicz MD}

Department of General,

Gastrointestinal and Endocrinological Surgery

Poznan University of Medical Sciences

Przybyszewskiego 49

60-355 Poznań, Poland

fax +48618691684

e-mail: tbanasiewicz@op.pl 